\title{
Beam Energy Scan Program at RHIC (BES I and BES II) - Probing QCD Phase Diagram with Heavy-Ion Collisions
}

\author{
Grazyna Odyniec ${ }^{1}$ (for the STAR Collaboration) \\ Lawrence Berkeley National Laboratory, Nuclear Science Division, Berkeley, \\ Berkeley, CA 94720, USA \\ E-mail: G_Odyniec@,lbl.gov
}

In 2010, a multi-step experimental program, the Beam Energy Scan (BES), was launched at the Relativistic Heavy Ion Collider (RHIC) to investigate the phase diagram of strongly interacting nuclear matter. The BES phase I (BES I) was completed in 2011 with $\mathrm{Au}+\mathrm{Au}$ data sets in energy range from $39 \mathrm{GeV}$ to $7.7 \mathrm{GeV}$. This was complemented by earlier $(62.4,130$ and $200 \mathrm{GeV})$ and later $(54.5 \mathrm{GeV})$ collected data sets of $\mathrm{Au}+\mathrm{Au}$ collisions. Many measurements taken by the STAR (Solenoidal Tracker At RHIC) detector at $\sqrt{S}_{\mathrm{s}}$ below the RHIC injection energy suffer from large statistical errors, sharply increasing with decreasing energy. Nevertheless, they allowed for the first time a direct study of the QCD critical point (CP) and phase transition signatures. The results of these studies are presented. In 2015, the Beam Energy Scan program was extended to energies below $\sqrt{\mathrm{s}} \mathrm{NN}=7.7 \mathrm{GeV}$ by successful implementation of the fixed-target mode of data taking (FXT) in the STAR experiment, in addition to the standard collider configuration. In the fixed target mode, ions circulating in one ring of the collider interact with a stationary target at the entrance of the STAR Time Projection Chamber. The first results from the exploratory FXT run with $\mathrm{Au}+\mathrm{Au}$ collisions at $\sqrt{\mathrm{s}}_{\mathrm{NN}}=4.5 \mathrm{GeV}$ are presented. The ongoing second phase of BES (BES II), which includes both collider and FXT data taking, will provide data sets with one-order-ofmagnitude larger statistics in collider mode, and with two-orders-of-magnitude larger statistics in FXT mode at each of several energies down to $\sqrt{\mathrm{s}} \mathrm{NN}=3.0 \mathrm{GeV}$.

Corfu Summer Institute 2018 "School and Workshops on Elementary Particle Physics and Gravity" (CORFU2018)

31 August - 28 September, 2018

\section{${ }^{1}$ Speaker}




\section{Introduction}

The first decade of RHIC operations has established that the highly excited, strongly coupled nuclear matter with partonic degrees of freedom (Quark Gluon Plasma, QGP) was created in $\mathrm{Au}+\mathrm{Au}$ collisions at $200 \mathrm{GeV}$ energy in the nucleon-nucleon center of mass.

The study of the QGP properties still continues. In parallel, in 2011, the search for signals of the anticipated transition from hadronic to partonic degrees of freedom, and back to the state of hadron gas, was initiated. Finding conditions at which thermodynamically distinct phases of nuclear matter occurred and/or coexist at equilibrium, i.e. locating the place of the phase transition and the position of the Critical Point of the QCD phase diagram, was undertaken by the BES program at RHIC.

While on the theoretical side there is still ongoing debate as to whether a CP exists at all, Lattice Gauge Theory calculations, which with time are becoming increasingly accurate, show that the cross-over region $\left(\mu_{\mathrm{B}} \sim 0\right)$ occurs at $\mathrm{T}_{\mathrm{c}}$ of about $154 \pm 9 \mathrm{MeV}$ [1]. Away from $\mu_{\mathrm{B}} \sim 0$ theoretical calculation are still unreliable and are struggling with difficulties.

The heavy ion experiments offer the unique and only way to study the nature of the QCD phase diagram as a function of chemical potential $\mu_{\mathrm{B}}$ and temperature $\mathrm{T}$. The initial conditions in the experiment can be varied by alternating the beam energy.

Figure 1 shows a generally accepted scheme of the QCD phase diagram: Temperature $\mathrm{T}$ vs chemical potential $\mu_{\mathrm{B}}$. At the top RHIC energies (T $\left.\sim 150-170 \mathrm{MeV}, \mu_{\mathrm{B}} \sim 0\right)$ QCD calculations predict $[2,3]$ smooth cross-over (top left of Fig.1), while at lower T and higher $\mu_{\mathrm{B}}$ the first order phase transition is expected [4,5] (right side of Fig.1). The end point of a first order phase transition line marks the QCD critical point - red circle in Fig.1.

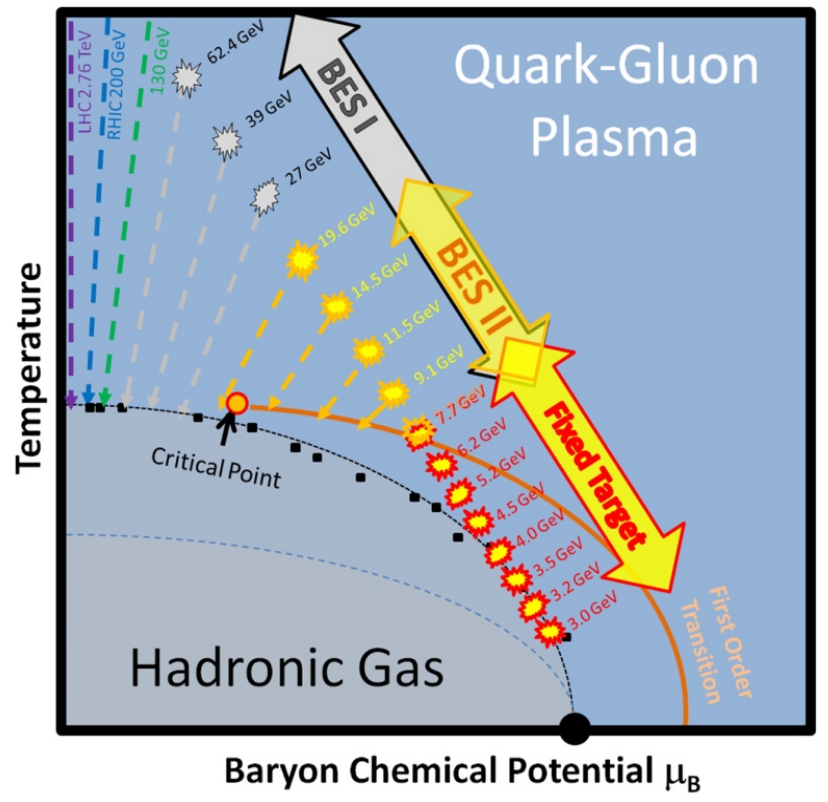

Figure 1. The schematic of the QCD Phase Diagram with shown coverage of the RHIC BES program. The red solid line represents phase boundary and the red circle a position of CP. Yellow lines represent possible reaction trajectories. 
The Beam Energy Scan program at RHIC, with variable beam energies, was able to scan $\mu_{\mathrm{B}}$ from $\sim 400 \mathrm{MeV}$ to $\sim 20 \mathrm{MeV}$. The first phase of BES included energies 7.7, 11.5, 14.5, 19.6, 27 and $39 \mathrm{GeV}$, which together with earlier $(62.4,130$ and $200 \mathrm{GeV})$ and later $(54.5 \mathrm{GeV})$ obtained data sets allowed BES to cover a remarkable $\mu_{\mathrm{B}}$ span of about $400 \mathrm{MeV}$. The choice of energies provided almost uniform coverage of unknown $\left(T, \mu_{\mathrm{B}}\right)$ and the results of analyses of data sets obtained at all these energy steps allowed narrowing down an area of interest for further study to be carried in the second phase of BES program.

In the next sections the specific goals of the BES program are discussed in more detail, together with the results of an analysis of data collected during the first phase of BES. The first results from the exploratory fixed-target run at energy of $4.5 \mathrm{GeV}$ are also discussed, as well as plans and expectations for the presently ongoing second phase of BES, which will consist of both (collider and fixed-target) modes of operation.

\section{BES at RHIC}

The main scientific goals of the BES program are as follows:

\subsection{The search for the onset of the deconfinement}

The scan of the phase diagram with variable collision energy (which represents steps in $\mu_{\mathrm{B}}$ ), from $200 \mathrm{GeV}$ down to a few $\mathrm{GeV}$, is expected to show the particular energy value at which the observed (at the top RHIC energies) QGP signatures will change or disappear. If such a change is observed, it might not necessarily signal that the system is back to a hadron gas phase. The change of one signature would not be enough because there are a number of other nondeconfinement phenomena which may cause such, or a similar, effect. Therefore, the study must include many relevant observables. The simultaneous changes observed in multiple studies addressing different QGP signatures would provide a compelling case.

\subsection{The search for the critical point of the QCD phase diagram.}

These studies are focused on finding critical fluctuations associated with the strong oscillations of susceptibilities predicted for vicinity of CP, which marks the end-point of the firstorder phase transition. Identification of such critical behavior would be a defining observation.

\subsection{The search for the signatures of the first-order phase transition.}

Due to the small size of the system, there is a concern that the finite size effects might modify the critical behavior. Therefore, the search for the softening of an Equation-Of-State (EOS) as a system enters unstable mixed phase region, implicitly associated with a crossing of the first-order phase transition, was proposed as a third goal of BES program.

\section{STAR experiment at RHIC}

The RHIC and the STAR experiment are ideal for these studies.

Even with the LHC running successfully at CERN, RHIC is the only facility capable of providing the wide range of beam energies necessary to execute the BES program. 
The STAR detector, one of two large experimental set-ups on the RHIC floor, with its large and uniform acceptance in rapidity, transverse momentum and azimuth across all energies studied in collider mode, its excellent particle identification capabilities and unprecedented good detector performance, is in a unique position to study the phase transition of nuclear matter.

In this analysis three subsystems were used for tracking and particle identification: Time Projection Chamber (TPC), Time-Of-Flight (TOF) detector and Barrel Electro-Magnetic Calorimeter (BEMC).

\section{Results}

Presented results of BES I analyses are grouped to represent examples of each of the three main goals of the program. The first section will discuss the conditions reached in the nuclear collisions at BES energies, and the remaining sections will discuss the results related to the outlined objectives.

\subsection{Global properties of created nuclear matter}

The numerous data sets collected during BES I allowed the addressing of bulk properties of the medium created in collisions. The detailed studies of the $\mathrm{p}_{\mathrm{T}}$ spectra at midrapidity and the kinetic and thermal freeze-out parameters' analyses extracted for the ${\sqrt{\mathrm{S}_{\mathrm{NN}}}}=7.7,11.5,19.6,27$, $39,62.4$ and $200 \mathrm{GeV}$ can be found in [6]. However, the question most worth pondering is related to the value/range of the chemical potential reached in these collisions, i.e. did collisions reach suitable value of $\mu_{\mathrm{B}}$, that is suggested by theory for these studies?

The integrated yields and $\mathrm{p}_{\mathrm{T}}$ spectra of hadrons provide information about the system at freeze-out. The state when the inelastic interactions among particles stop, and the yields of the produced particles became fixed, is referred to as chemical freeze-out. Statistical thermal models well describe the chemical freeze-out stage with two main parameters: chemical temperature $T_{\text {ch }}$ and baryon chemical potential $\mu_{\mathrm{B}}[7,10,11,12,13]$.
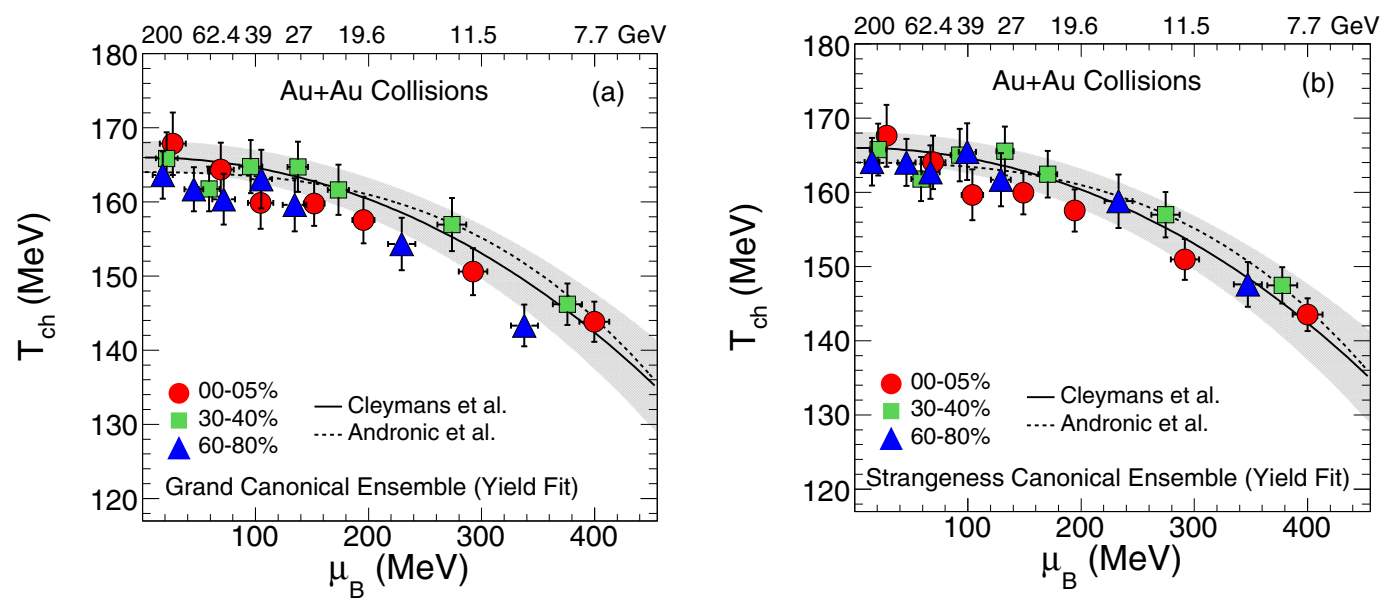

Fig.2 Chemical freeze-out temperature $T_{c h}$ vs baryon chemical potential $\mu_{\mathrm{B}}[6]$ fitted with the (a) Grand Canonical Ensemble and (b) Strangeness Chemical Ensemble in THERMUS model [7] using available particle ratios from BES I data sets (see energy scale at the top of figures). The lines represent two model predictions $[8,9]$. The grey bands represent the theoretical prediction ranges for [8]. The error bars are systematic. 
Figure 2 shows the centrality dependence of the chemical freeze-out temperature as a function of chemical potential based of THERMUS model [7] with (a) Grand Canonical Ensemble

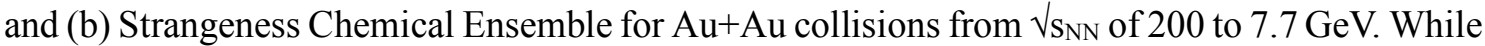
there are no significant changes in $\mathrm{T}_{\text {ch }}\left(\mathrm{T}_{\text {ch }}\right.$ decrease from $\sim 165 \mathrm{MeV}$ at $200 \mathrm{GeV}$ to $\sim 150 \mathrm{MeV}$ at $7.7 \mathrm{GeV}$ ), the chemical potential changes dramatically, from $\sim 20 \mathrm{MeV}$ at $200 \mathrm{GeV}$ to $\sim 400$ $\mathrm{MeV}$ at $7.7 \mathrm{GeV}$. Since the critical region is expected to extend to about $100 \mathrm{MeV}$ in $\mu_{\mathrm{B}}$ [14] around the $\mathrm{CP}$, the BES program sensitivity would reach to even higher $\mu_{\mathrm{B}}$ values, perhaps up to $\sim \mu_{\mathrm{B}}$ of $500 \mathrm{MeV}$. This demonstrates that the span of BES energies allows the reaching of the most desired, according to theory, $\mu_{\mathrm{B}}$ region.

\subsection{Disappearance of the QGP signals}

The data collected by BES I clearly shows that observables are evolving strongly with energy. The results at the top RHIC energies indicate the formation of the phase with partonic degrees of freedom, which is expected to change and /or disappear with the lowering of collision energy (increasing chemical potential $\mu_{\mathrm{B}}$ ) as system returns to the hadron gas phase. A broad range of BES energies allows the following of the evolution of the QGP signatures and to test this hypothesis. One of a generally accepted QGP signatures is a scaling of an elliptic flow $\left(\mathrm{v}_{2}\right)$ with the Number of Constituent Quarks (NCQ scaling) of a given hadron (2 for mesons and 3 for baryons). This scaling indicates that elliptic flow is acquired in a very early stage of the collision where quarks are relevant degrees of freedom. In Fig.3, the $\mathrm{p}_{\mathrm{T}}$ dependence of the proton and antiproton $\mathrm{V}_{2}$ is shown for minimum bias $\mathrm{Au}+\mathrm{Au}$ at ${\sqrt{\mathrm{S}_{\mathrm{NN}}}}=7.7,11.5,27$ and $39 \mathrm{GeV}$. At all energies $\mathrm{v}_{2}$ gradually increases with energy and $\mathrm{p}_{\mathrm{T}}[14]$.

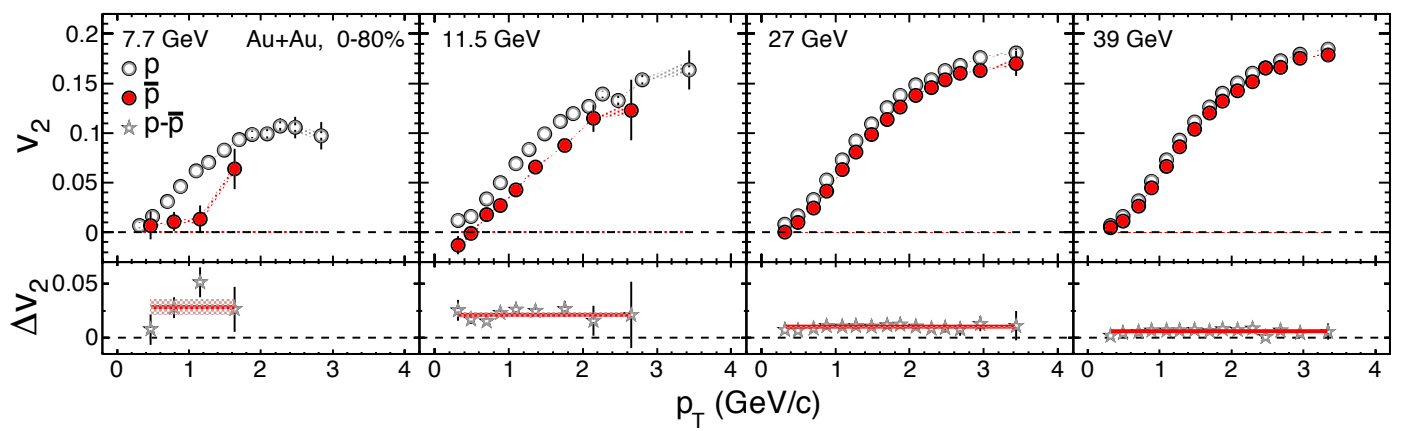

Fig. 3 The elliptic flow v2 of protons and anti-protons as a function of the transverse momentum, $\mathrm{p}_{\mathrm{T}}$, for $0-80 \%$ central $\mathrm{Au}+\mathrm{Au}$ collision [14]. The lower panels show the difference in $\mathrm{v}_{2}\left(\mathrm{p}_{\mathrm{T}}\right)$ between the particles and anti-particles. The solid curves represent fit results with a horizontal line.

At $39 \mathrm{GeV}$ the proton $\mathrm{v}_{2}$ is only slightly higher than the anti-protons $\mathrm{v}_{2}$, but this difference increases substantially with decreasing beam energy. The lower panel of Fig. 2 shows that the difference in $\mathrm{v}_{2}$ for proton and anti-proton does not depend on $\mathrm{p}_{\mathrm{T}}$, as demonstrated by the horizontal line. The $\mathrm{v}_{2}\left(\mathrm{p}_{\mathrm{T}}\right)$ behavior for other particles is similar to that for protons and antiprotons. The $\mathrm{v}_{2}$ vales are found to be larger for particles than for anti-particles, except pions for which the opposite ordering is observed. Figure 4 summarizes the variation in $\mathrm{v}_{2}$ between particles

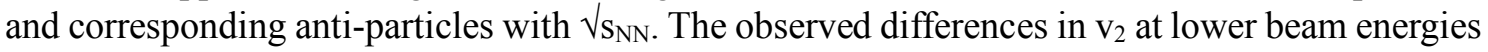
demonstrate that the number of constituent quarks (NCQ) scaling between particles and antiparticles observed at ${\sqrt{\mathrm{S}_{\mathrm{NN}}}}=200 \mathrm{GeV}$ is no longer valid at lower energies. The lower the energy the stronger the violation of NCQ scaling is observed. The breaking of the NSQ scaling could 
indicate increased contribution from hadronic interactions in the system evolution with decreasing energy

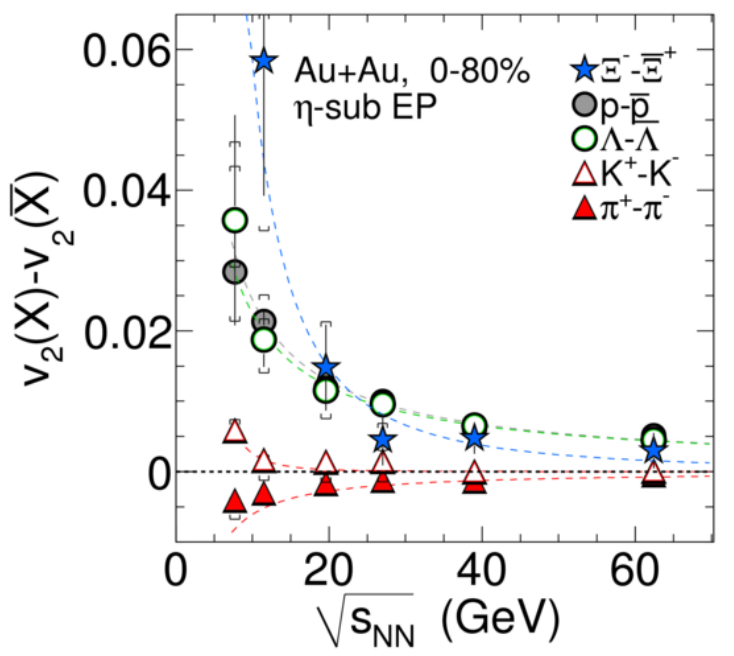

Fig. 4 The difference in $\mathrm{v}_{2}$ between particles and their corresponding anti-particles (see text) as a function of ${\sqrt{\mathrm{S}_{\mathrm{NN}}}}_{\text {for }} 0-80 \%$ central $\mathrm{Au}+\mathrm{Au}$ collisions [14]. The dash lines represent fit results with the power-law function. The error bars represent combined statistical and systematic errors.

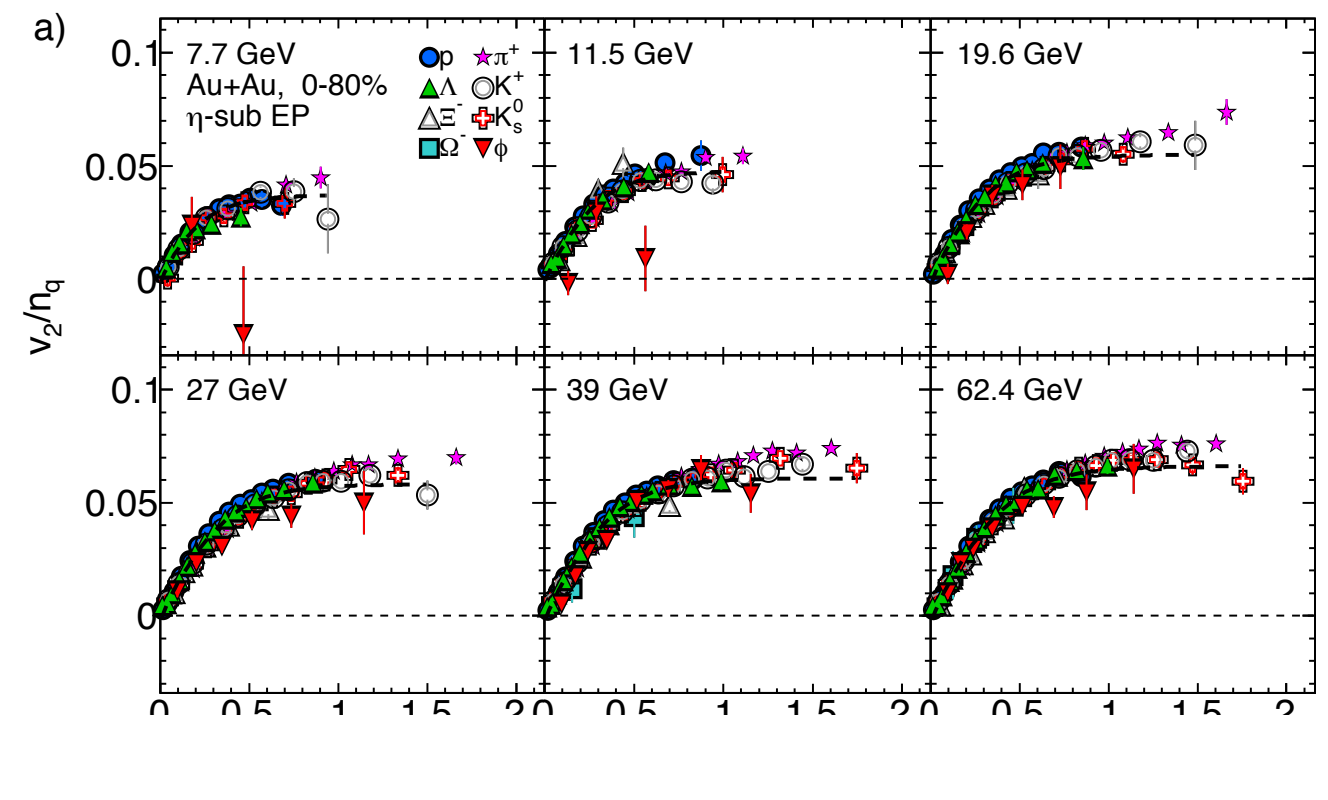

Fig.5 The NCQ scaled elliptic flow, $\mathrm{v}_{2} / \mathrm{n}_{\mathrm{q}}$ vs $\left(\mathrm{m}_{\mathrm{t}}-\mathrm{m}_{0}\right) / \mathrm{n}_{\mathrm{q}}$, for $0-80 \%$ central $\mathrm{Au}+\mathrm{Au}$ collisions for selected particles [14]. Note the different behavior of $\phi \mathrm{v}_{2}$ at $7.7 \mathrm{GeV}$ and $11.5 \mathrm{GeV}$.

The elliptic flow of $\phi$ is particularly interesting as it is unlikely to be built up during the hadronic phase of the collision because of its low scattering cross section. Figure 5 shows the elliptic flow, $\mathrm{v}_{2} / \mathrm{n}_{\mathrm{q}} \mathrm{vs}\left(\mathrm{m}_{\mathrm{t}}-\mathrm{m}_{0}\right) / \mathrm{n}_{\mathrm{q}}$ for $0-80 \%$ central $\mathrm{Au}+\mathrm{Au}$ collisions for selected particles, among others also for $\phi$. At ${\sqrt{\mathrm{S}_{\mathrm{NN}}}} 7.7 \mathrm{GeV}$ and $11.5 \mathrm{GeV}$ the measured elliptic flow of $\phi$ is consistent with zero, although with large errors [14]. Measured $\mathrm{v}_{2} \cong 0$ of $\phi$ meson at lower energies, while non- 
zero values are observed for other species, is suggestive of a fireball that is no longer in the QGP phase below $\sim{\sqrt{\mathrm{S}_{\mathrm{NN}}}}=15 \mathrm{GeV}$.

Another example of the disappearance of the standard QGP signature is the strong suppression of high $\mathrm{p}_{\mathrm{T}}$ charge particles ( $\sim$ jet quenching), as indicated by binary scaled yields, which is no longer present in the collisions of the BES lower energies for any centrality [15]

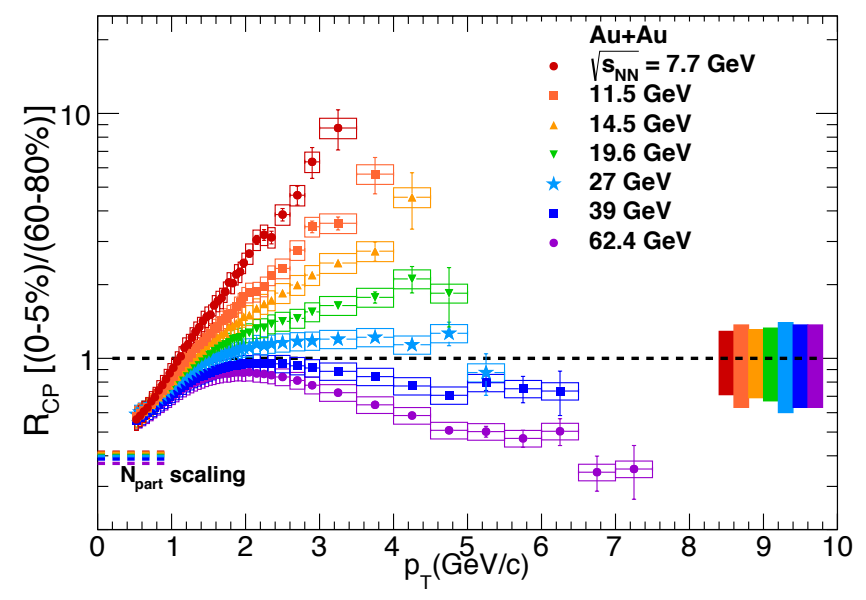

Fig.6 Charged hadron $\mathrm{R}_{\mathrm{cp}}$ for RHIC BES energies [15]. The error bands at unity on the right side of the plot indicate $\mathrm{p}_{\mathrm{T}}$ independent systematic uncertainty in $\mathrm{N}_{\mathrm{bin}}$ scaling with the color in the band corresponding to the color of the data points for that energy. The vertical error bars correspond to statistical errors.

Figure 6 shows the $\mathrm{R}_{\mathrm{cp}}$ of charged hadrons from $\mathrm{Au}+\mathrm{Au}$ collisions at ${\sqrt{\mathrm{S}_{\mathrm{NN}}}}=7.7,11.5$, $14.5,19.6,27$ and $39 \mathrm{GeV}$ together with the points for $62.4 \mathrm{GeV}$. The $\mathrm{p}_{\mathrm{T}}$ reach extends beyond 3 $\mathrm{GeV} / \mathrm{c}$ for all hadrons at all BES energies. The figure shows dramatic change in the suppression pattern, and while there is clear evidence of suppression at ${\sqrt{\mathrm{S}_{\mathrm{NN}}}}=39$ and $62.4 \mathrm{GeV}$, the lower energies $\left({\sqrt{\mathrm{S}_{\mathrm{NN}}}}=27 \mathrm{GeV}\right.$ and $\left.19.6 \mathrm{GeV} \mathrm{GeV}\right)$ show a leveling out, and the lowest energies $\left({\sqrt{\mathrm{S}_{\mathrm{NN}}}}\right.$ $=7.7,11.5$ and $14.5 \mathrm{GeV}$ ) even an enhancement.

Word of caution: even at energies and centralities where this signature seems to be lost, a QGP can still be formed since competing phenomena responsible for enhancement (e.g. Cronin effect) may overwhelm the suppression from energy loss.

Another interesting piece of information comes from the measurement of triangular flow, $\mathrm{v}_{3}$, of charged particles [16]. Models predict that the development of triangular flow can only take place in the presence of a low viscosity plasma phase. The measured value of $\mathrm{v}_{3}$ is non-zero for central events at all BES energies, i.e. it persists for collisions with $\mathrm{N}_{\text {part }}>50$ down to the lowest energies, while for collisions with $\mathrm{N}_{\text {part }}<50$ disappears for energies below $14.5 \mathrm{GeV}$. That might be interpreted as evidence for some presence of the plasma phase in the events of sufficiently high centrality even at the lowest energies of the scan.

All these observed changes in behavior, together with the others discussed broadly in [17] are localized at the lower part of the energy span covered by BES, suggesting that the phase transition between partonic and hadronic states might take place just there, below ${\sqrt{\mathrm{s}_{\mathrm{NN}}}}$ of $19 \mathrm{GeV}$. 


\subsection{The Critical Point search}

The observations discussed in the previous section suggest the possibility of the existence of the critical point. The Lattice and phenomenological calculations show that [17] the presence of CP might result in a divergence of thermodynamical susceptibilities and correlation length, what translates to non-monotonic behavior of correlations and fluctuations related to the conserved quantities, such as baryon number (B), charge (Q) and strangeness (S) [18], which could be observed and measured experimentally. The higher moments of these distributions (B, $\mathrm{Q}, \mathrm{S}$ ) are predicted to have increased sensitivity to critical phenomena, e.g. the second moment (variance) is proportional only to the square of the correlation length $(2-3 \mathrm{fm})$ while the third moment, the skewness $\mathrm{s} \sim \xi^{4.5}$ and the forth moment, the kurtosis $\kappa \sim \xi^{7}$ [18]. Note, the direct relation between moments of event-by-event distributions of the conserved quantities $(B, Q, S)$ and thermodynamical susceptibilities computed on Lattice QCD, e.g. $\kappa \sigma^{2}\left(\sigma^{2}\right.$ is a variance and $\kappa$ is the kurtosis) can be expressed as the ratio of susceptibilities $\chi^{(4)} / \chi^{(2)}$. Predictions are that the behavior of fluctuations and correlations in the deconfined phase are different than in the hadron gas, therefore the stress of the BES program was put on the search for discontinuities in fluctuations and correlations as a function of ${\sqrt{\mathrm{S}_{\mathrm{NN}}}}$ (i.e. as a function of $\mu_{\mathrm{B}}$ ). The excitation function for net-proton high moments $\left(\kappa \sigma^{2}\right)$ in the $5 \%$ most central $\mathrm{Au}+\mathrm{Au}$ collisions, for three centrality bins $0-5 \%, 5-10 \%$ and $70-80 \%$, is presented in Fig.7 [19] (note, that Fig.7 shows net-proton kurtosis results for the extended $\mathrm{p}_{\mathrm{T}}$ range up to $2 \mathrm{GeV} / \mathrm{c}$, the earlier STAR results have had a cut on $0.8 \mathrm{GeV} / \mathrm{c})$.

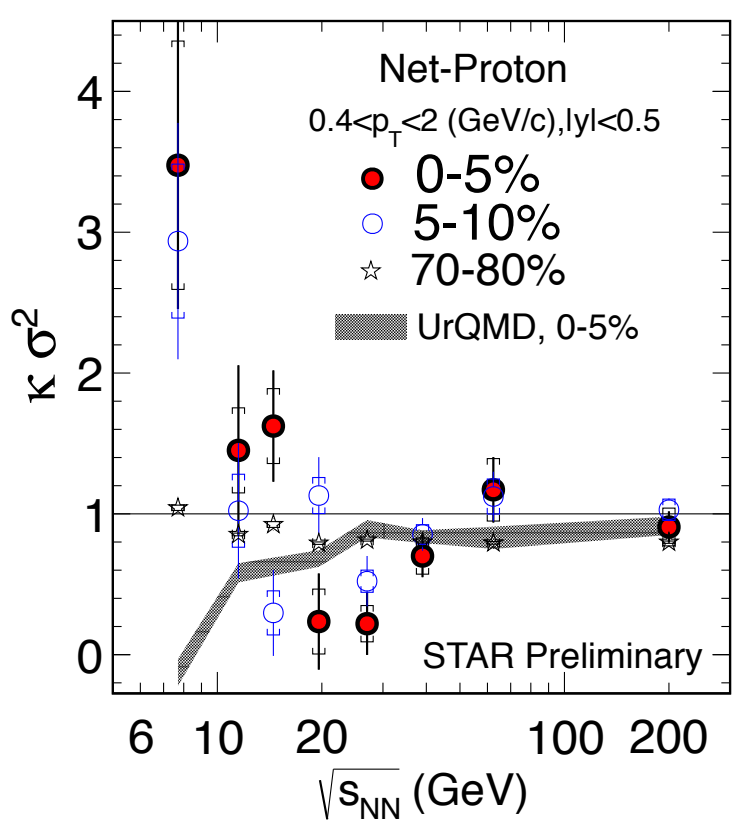

Fig.7 Colliding energy and centrality dependence of the net-proton $\kappa \sigma^{2}$ in three bins of centrality $(0-5 \%, 5-10 \%, 70-80 \%)$ in $\mathrm{Au}+\mathrm{Au}$ collisions at RHIC. The black band shows UrQMD transport model calculations for the most central bin $0-5 \%$.

Figure 7 (taken from [38]) shows that non-monotonic behavior is clearly present in the most central $(0-5 \%)$ bin at the lower part of the energy range what is consistent with the expected behavior near a critical point, while the peripheral collisions exhibit a very smooth trend. The UrQMD transport model calculations [21], which do not include critical point, show suppression at low energies which is attributed to the baryon number conservation. At the CPOD conference in 2015 it was shown that these measurements are qualitatively consistent with a QCD based model which includes a critical point [20]. Away from CP those enhanced fluctuations are 
expected to go down $[17,22]$. There is no data to test this. The only available data at lower energies $\left({\sqrt{\mathrm{S}_{\mathrm{NN}}}}=2.4 \mathrm{GeV}\right)$ is from the HADES experiment [23], but these results can not be used for direct comparison because they cover a different centrality, $\mathrm{p}_{\mathrm{T}}$ and rapidity range. The presently ongoing run of the second phase of BES, which in addition to the collider mode is also running FXT mode, will allow the study moments at energies as low as ${\sqrt{\mathrm{S}_{\mathrm{NN}}}}=3 \mathrm{GeV}$, and address the discussed change in fluctuations.

Contrary to the non-monotonic behavior of net-proton $\kappa \sigma^{2}$, moments of net-charge and net-kaon (representing net-strangeness) distributions studies across all BES energies do not show any non-monotonic behavior at the current precision level.

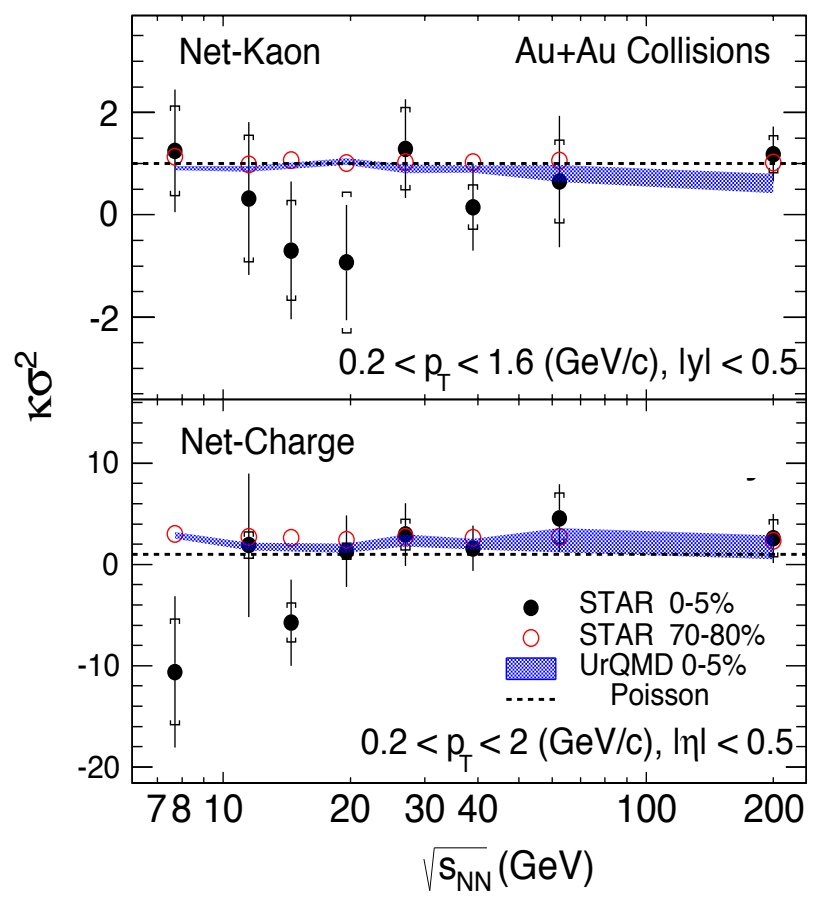

Fig 8. Forth moment of net-kaon and net-charge distributions in $\mathrm{Au}+\mathrm{Au}$ collisions at all BES energies [24].

Figure 8 shows $\kappa \sigma^{2}$ of net- kaon and net-charge distributions [24], which are both consistent with unity, albeit with large errors. The blue band represents the UrQMD transport model with "non-CP scenario", which shows no energy dependence.

Very recently STAR collaboration published [39] the first measurements of a complete second-order cumulant matrix of net-charge, net-proton and net-kaon multiplicity distributions for the first phase of BES program. Model calculations based on thermal (HRG) and non-thermal (UrQMD) production of hadrons can not describe the data - see contribution by Arghya Chatterjee in this proceeding.

\subsection{Search for the first-order phase transition}

The directed flow $\mathrm{v}_{1}$, which is the first harmonic coefficient in the Fourier expansion of the final-state particles azimuthal distribution, describes their sideward motion. Hydrodynamical and transport models show that $\mathrm{v}_{1}$ is sensitive to details of the expansion and compression during early stages of the collision and also to the first-order phase transition. The proposed signature [25] is the double change of the sign (dip) in the slope of $\mathrm{dv}_{1} / \mathrm{dy}$ for protons in function of energy, 
which would signal the softening of the equation-of-state (EOS), and therefore the first-order phase transition.

The direct flow excitation function for protons, anti-protons and their properly scaled difference (net-protons) in $\mathrm{Au}+\mathrm{Au}$ collisions is presented in Fig. 9. The lower panel in Fig.9 shows [19] that the $\mathrm{dv}_{1} /$ dy of net-protons at midrapidity changes its sign twice between ${\sqrt{\mathrm{S}_{\mathrm{NN}}}}=7.7-39$ $\mathrm{GeV}$. The prominent dip and its associated $\mathrm{dv}_{1} / \mathrm{dy}$ double sign change bear a striking resemblance to the predicted signature of the softening of the EOS [25]. Note that the observed experimentally dip is at a much higher value of ${\sqrt{S_{N N}}}_{\text {than }}$ model predictions. There have been several recent $\mathrm{v}_{1}(\mathrm{y})$ model calculations with various assumptions regarding QCD equation of state, but the predicted values of $v_{1}$ are strongly sensitive to the models' details [26].

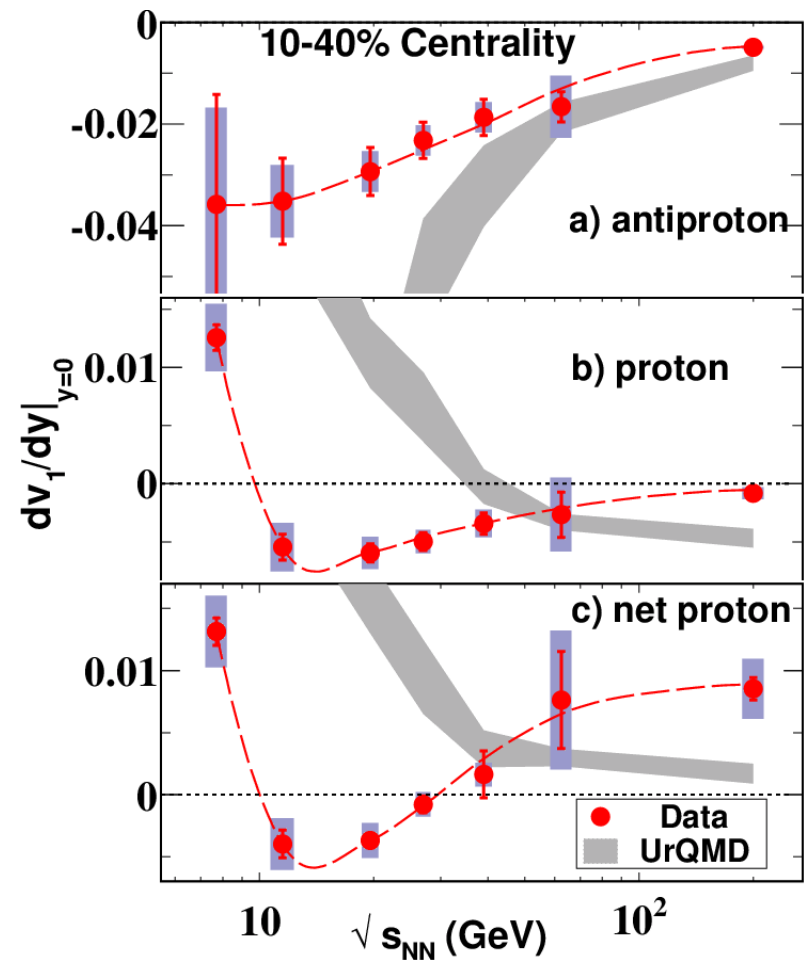

Fig.9 The $\mathrm{dv}_{1} / \mathrm{dy}$ of protons, anti-protons and net-protons in $\mathrm{Au}+\mathrm{Au}$ collisions at BES energies [19]. The gray band representing UrQMD transport model shows a positive slope for the $\mathrm{dv}_{1} / \mathrm{dy}$ of net-protons at all energies.

None of the models can reproduce the minimum in $\mathrm{dv}_{1} / \mathrm{dy}$ over the observed energy range and the assumption of purely hadronic physics seems to be disfavored [19].

Recently STAR reported the first measurements of directed flow for $\Lambda$, anti- $\Lambda, \mathrm{K}+/-, \mathrm{K}^{0}$ and $\phi$ in $\mathrm{Au}+\mathrm{Au}$ collisions at eight BES energies from 7.7 to $200 \mathrm{GeV}$ [27].

The net-proton $\mathrm{v}_{1}$ is a result of complex interplay between $\mathrm{v}_{1}$ of baryons transported from beam and $\mathrm{v}_{1}$ from pair production. The net-proton $\mathrm{v}_{1}$, which represents the directed flow of transported baryons is shown in Fig. 10. The $v_{1}$ of net- $\Lambda$ hyperons has a similar behavior [27].

The double-sign change prominent in $\mathrm{dv}_{1} / \mathrm{dy}_{\text {of }}$ net-protons and net-lambdas is not present in $\mathrm{dv}_{1} / \mathrm{dy}$ of kaons. These results are not yet reproduced by theory. 


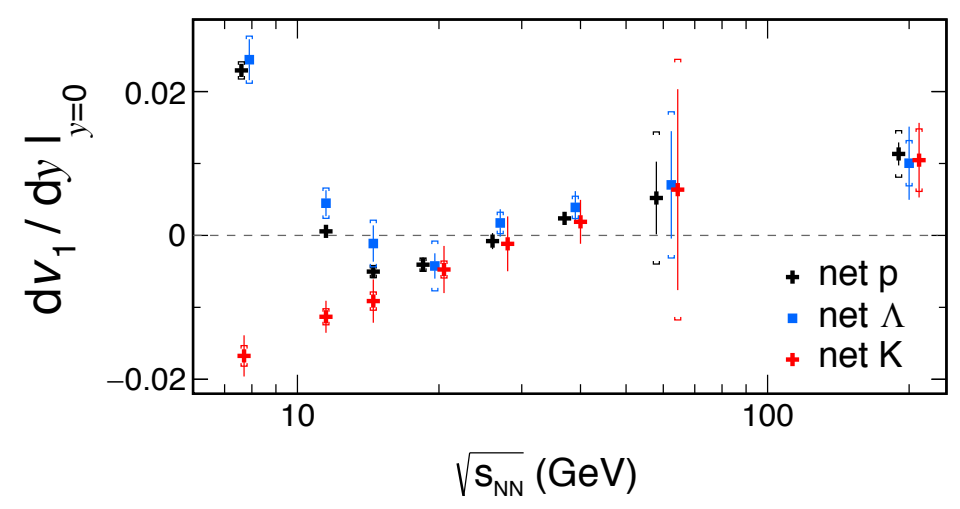

Fig. $10 \mathrm{The}_{\mathrm{dv}} / \mathrm{dy}$ for the net-protons, net- $\Lambda$ and net-kaons versus energy [27].

The present energy-dependent measurements will be greatly enhanced during STAR BES II run (presently ongoing) with increased statistics, allowing to analyze data in rapidity bins and therefore addressing properly influence of the nuclear stopping power in these collisions.

The higher harmonics in Fourier decomposition received recent attention after the importance of initial density fluctuations was realized [29]. When divided by multiplicity, $\mathrm{v}_{3}$ shows a local minimum in the region ${\sqrt{\mathrm{S}_{\mathrm{NN}}}}$ of $15-20 \mathrm{GeV}$. This has not been shown by any model and can indicate an interesting trend in the pressure gradient developed inside a system.

\subsection{Summary of BES I findings}

The BES I data analysis extended the $\mu_{\mathrm{B}}$ reach of RHIC to the value of approximately $400 \mathrm{MeV}$. The addition of the exploratory test run with the fixed-target mode of operation allowed STAR to extend $\mu_{\mathrm{B}}$ range even further, to values of about $700-800 \mathrm{MeV}$. Both RHIC and STAR demonstrated excellent performance at all energies run. Very interesting results include:

- Several signatures demonstrated the dominance of parton regime at the BES highest energies. These signatures either disappeared, lost significance, or lost sufficient reach in the low energy region of the scan (NCQ scaling, $\phi \mathrm{v}_{2}$, high $\mathrm{p}_{\mathrm{T}}$ suppression, ...) indicating that the hadronic interactions became dominant at lower energies

- Both net-proton and net- $\Lambda$ showed a double sign change in mid-rapidity $\mathrm{dv}_{1} / \mathrm{dy}$, as predicted for the possible first order phase transition, indicating the softening of EOS around $11.5-19.6 \mathrm{GeV}$

- Non-monotonic energy dependence of the $\kappa \sigma^{2}$ net-proton correlation function suggests the signs of critical fluctuations

The implications of these measurements in understanding the QCD phase structure, while broadly discussed in the community, are not yet resolved. 


\section{Fixed-target mode}

The data clearly shows that observables are evolving strongly with energy at the lowest energies accessible in collider mode at RHIC.

While continued theoretical effort is not yet able to explain the origins of the evolution, it is crucial to precisely map and quantitatively understand the behavior at lower energies. RHIC can not provide adequate luminosity at energies below $7.7 \mathrm{GeV}$ in the collider mode of operation, therefore STAR has initiated the fixed-target (FXT) program. An internal stationary target at the entrance of the STAR Time Projection Chamber, $\sim 2 \mathrm{~m}$ from the center of the detector, was installed and ions circulating in only one ring of the collider were used for collisions. The first exploratory run with $\mathrm{Au}$ ions at injection energy and the stationary $\mathrm{Au}$ target provided $\mathrm{Au}+\mathrm{Au}$ collisions at ${\sqrt{\mathrm{S}_{\mathrm{NN}}}}=4.5 \mathrm{GeV}$. Similar energies were studied in the $1990 \mathrm{~s}$ during the heavy-ion program at the Alternating Gradient Synchrotron (AGS), with top energy of ${\sqrt{\mathrm{S}_{\mathrm{NN}}}}=4.9 \mathrm{GeV}$. So, the data obtained with the STAR FXT program can be checked against earlier obtained AGS results, providing validation for the FXT program. The $4.5 \mathrm{GeV} \mathrm{Au}+\mathrm{Au}$ data from STAR FXT run represents the first point at so low energies in the new precision map of QCD phase diagram.

In this chapter the preliminary results for $\mathrm{Au}+\mathrm{Au}$ collisions at ${\sqrt{\mathrm{S}_{\mathrm{NN}}}}=4.5 \mathrm{GeV}$ are presented, including the directed and elliptic flow of identified hadrons [40] and femtoscopy radii for pions.
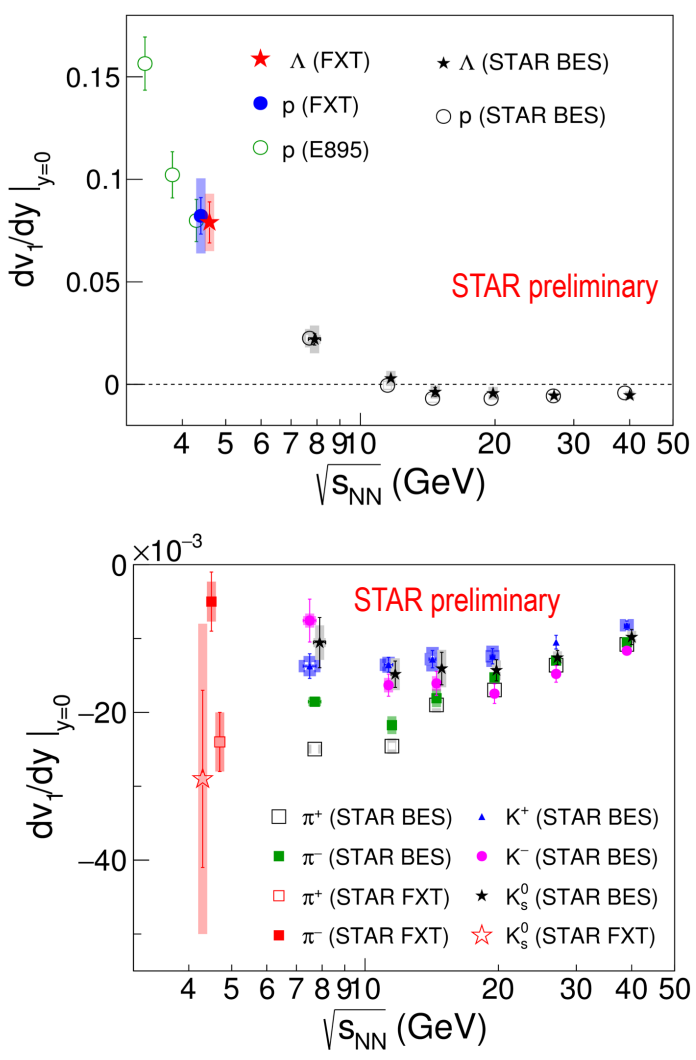

Fig.11 Beam energy dependence of directed flow $\mathrm{dv}_{1} / \mathrm{dy}$ at midrapidity for baryons (upper plot) and mesons (lower plot) measured by several experiments [26, 28, 29]. FXT points [40] are slightly offset horizontally. 
Figure 11 presents slopes $\mathrm{dv}_{1} / \mathrm{dy}$ of midrapidity for protons, lambdas, kaons and pions measured in $\mathrm{Au}+\mathrm{Au}$ collisions in FXT mode at ${\sqrt{\mathrm{S}_{\mathrm{NN}}}}=4.5 \mathrm{GeV}$. The proton $\mathrm{v}_{1}(\mathrm{y})$ slope measurement at ${\sqrt{\mathrm{S}_{\mathrm{NN}}}}=4.5 \mathrm{GeV}$ lies within errors on a smooth interpolation between the same observables from STAR's beam energy scan in collider mode [19] and E895 [29]. The highest E895 energy point at ${\sqrt{\mathrm{S}_{\mathrm{NN}}}}=4.3 \mathrm{GeV}$ agrees with the current FXT measurement well within uncertainties. Proton and $\Lambda$ directed flow agree within errors at ${\sqrt{\mathrm{S}_{\mathrm{NN}}}}=4.5 \mathrm{GeV}$, a pattern also observed by STAR at ${\sqrt{\mathrm{S}_{\mathrm{NN}}}}=7.7 \mathrm{GeV}$ and above [27].

The elliptic flow of identified particles (protons and pions) in $\mathrm{Au}+\mathrm{Au}$ at ${\sqrt{\mathrm{S}_{\mathrm{NN}}}}=4.5 \mathrm{GeV}$ was studied, and while the elliptic flow of protons is compared with earlier AGS data with very good agreement (Fig.12), the elliptic flow of pions is totally new piece of information.

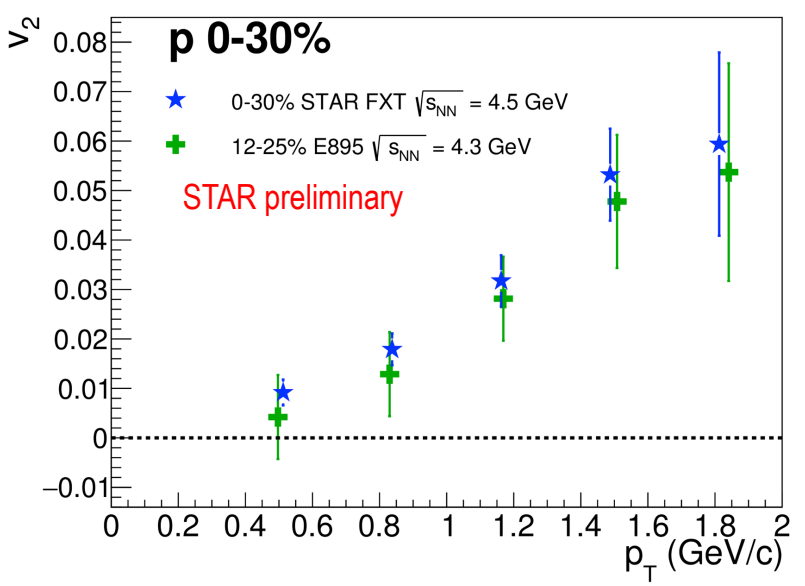

Fig. 12 Measured $v_{2}$ of protons. Blue stars are STAR FXT data (0-30\% centrality) and green crosses are E895 data (12-25\% centrality) [30].

Pion elliptic flow is published for the first time and compared with results at high energies. Both positive and negative charged pions are analyzed together. Figure 13 shows the excitation function of $\mathrm{v}_{2}$. The current results are consistent with trends established by previously published data.

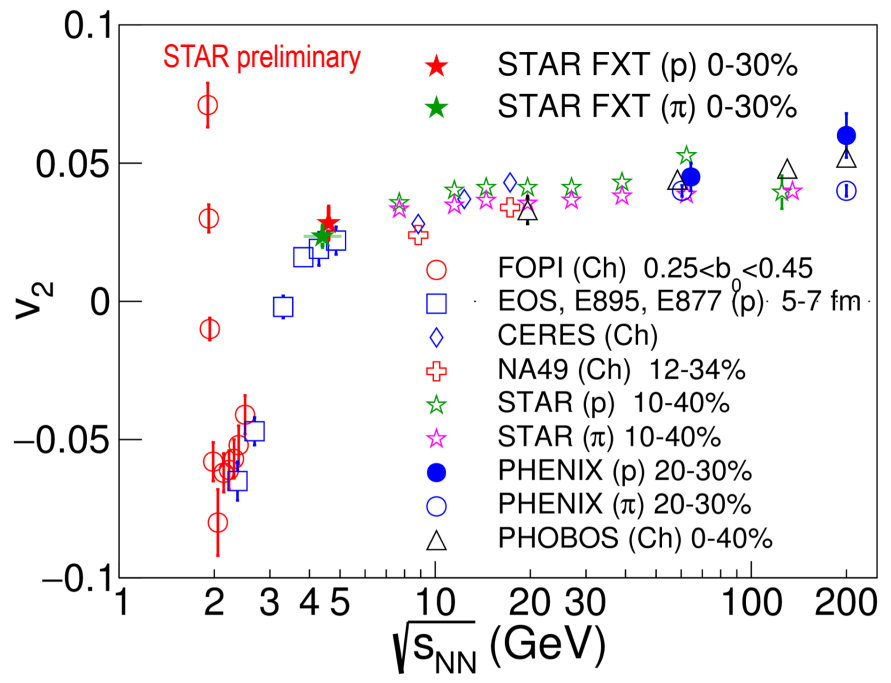


Fig. 13 The excitation function of charged particles $\mathrm{v}_{2}$ measured by several experiments. The STAR FXT point is near the region where a change of slope occurs.

Another confirmation of agreement with the earlier results comes from HBT analysis. Figure 14 shows the excitation function of the three HBT radii $\left(R_{\text {out }}, R_{\text {side }}\right.$ and $\left.R_{\text {long }}\right)$ for the STAR FXT point at ${\sqrt{\mathrm{S}_{\mathrm{NN}}}}=4.5 \mathrm{GeV}$, and E895 [31] and E866 experiments [32]. The $\mathrm{R}_{\text {side }}$ radius primarily reflects the spatial size of the pion emitting source, whereas $\mathrm{R}_{\text {out }}$ convolved this with the emission duration of the fireball [33]. The E895 and E866 points show a monotonically decreasing beam energy dependence. The fixed-target STAR points are consistent with this trend within errors.

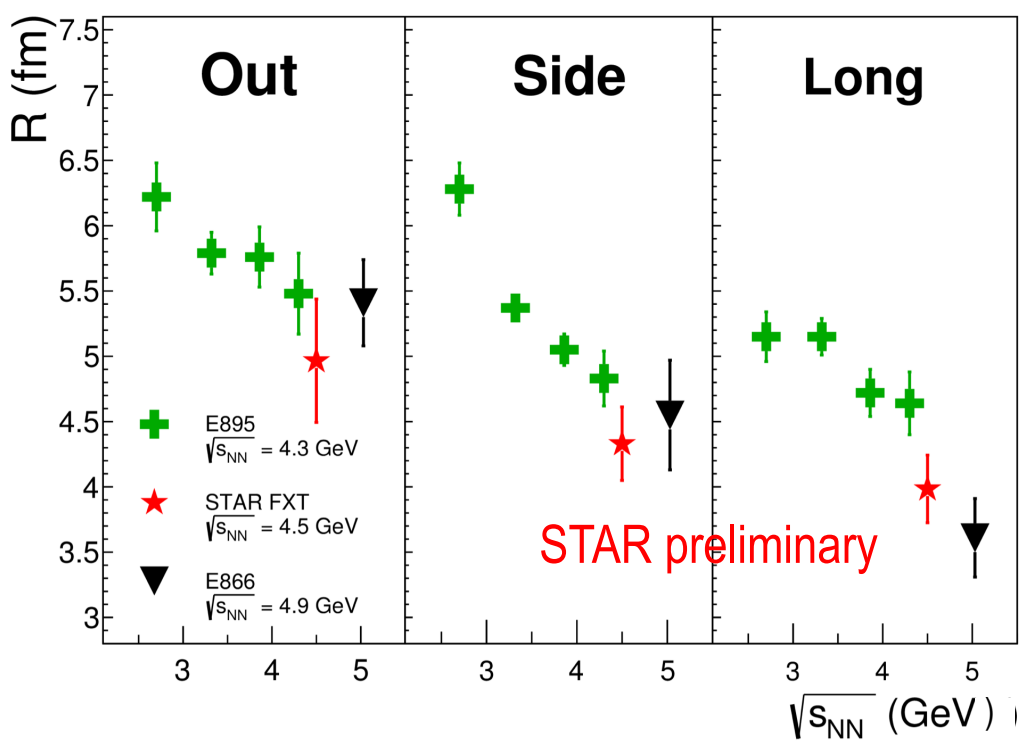

Fig.14 Excitation function of $\mathrm{R}_{\text {out }}, \mathrm{R}_{\text {side }}$ and $\mathrm{R}_{\text {long }}$ for three experiments: E895 [31], STAR and E866 [32]. STAR points show systematic errors only, as statistical errors are smaller than symbol size. Errors for E895 and E866 are statistical only.

Summarizing the first FXT run outcome: the ${\sqrt{\mathrm{S}_{\mathrm{NN}}}}=4.5 \mathrm{GeV}$ data on proton flow and pion femtoscopy agree quantitively with earlier measurements at similar energies. This provide crucial confidence in the data systematics produced by the STAR experiment in the novel fixedtarget configuration.

The directed flow $\mathrm{v}_{1}$ of protons and $\Lambda \mathrm{s}$ is strong and consistent with existing systematics in lower and higher energies. This is particularly important measurement as $\mathrm{v}_{1}$ of protons is one of the most intriguing BES I results. Both elliptic flow and HBT results agree with the earlier trends established by AGS. Pion $\mathrm{v}_{2}$ are measured for the first time at these energies. program.

Present measurements justified the inclusion of the FXT configuration into the BES II

\section{Beam Energy Scan II}

BES I data shows that RHIC "sits" at a "sweet spot" in energy, in which rapid changes occur in a number of signatures for energies up to approximately $20 \mathrm{GeV}$, while remaining surprisingly stable over two orders of magnitude to the LHC. Reported observations, although surely compelling physics-wise, suffer from large systematic errors and inadequate statistics. 
These results, however, provided a clear direction for the second part of the BES program with far better statistics thanks to improvements provided by the electron cooling upgrade of RHIC [34] and detector upgrades implemented in STAR experiment, allowing for much larger detector acceptance and excellent performance of the detector.

Additionally, the $4^{\text {th }}$ goal was added to the list of major objectives of the BES program. Namely, based on somewhat limited statistics, it was realized that the study of the chiral symmetry restoration, which may affect the mass and width of the $\rho(770), \omega(782)$ and $\phi(1020)$ vector mesons, can be undertaken via dilepton decays.

Both RHIC and STAR are well prepared for the challenges of BES II.

The three major upgrades are implemented into STAR experimental set-up:

- The inner TPC (iTPC) upgrade [35], i.e. continues instrumentation of the inner parts of TPC sectors, extends the pseudo-rapidity $\eta$ coverage from $(-1,1)$ to $(-1.5,1.5)$, which also allows for lowering of the $\mathrm{p}_{\mathrm{T}}$ cut-off from $\sim 125 \mathrm{MeV} / \mathrm{c}$ to $\sim 60 \mathrm{MeV} / \mathrm{c}$. It also provides improvement in $\mathrm{dE} / \mathrm{dx}(\sim 25 \%)$ and in momentum resolution $(\sim 15 \%$ at large momentum)

- The Event Plane Detector (EPD) upgrade [36], i.e. two azimuthally symmetric scintillator telescopes are placed symmetrically to both sides of the center of the detector along the beam direction, which improves event plane resolution by a factor of 2 approximately in $\mathrm{Au}+\mathrm{Au}$ collisions at ${\sqrt{\mathrm{S}_{\mathrm{NN}}}}=19.6 \mathrm{GeV}$, and a factor of 3 approximately at ${\sqrt{\mathrm{S}_{\mathrm{NN}}}}=7.7 \mathrm{GeV}$.

- $\quad$ The eTOF (end cap TOF) [37], joint project of STAR and CBM collaborations, which covers the pseudo-rapidity region of $(1.1,1.6)$ and improves particle identification in $\eta$ acceptance region added by the iTPC. This will particularly benefit FXT data.

RHIC is in the process of developing and testing the Low-Energy RHIC Electron Cooler (LEReC) for beams at ${\sqrt{\mathrm{S}_{\mathrm{NN}}}}=11.5 \mathrm{GeV}$ and below (to be run in 2020 and 2021) to increase the luminosity by a factor of 4 . Above ${\sqrt{\mathrm{S}_{\mathrm{NN}}}}=14.5 \mathrm{GeV}$, the RHIC luminosity increase of about a factor of 3 is due to improvements in bunch structure and $\beta^{*}$.

The Beam Energy Scan Program Phase II with $\mathrm{Au}+\mathrm{Au}$ collisions began in February of 2019. It will extend the reach of the STAR experiment across an important energy regime of high baryon densities, from ${\sqrt{\mathrm{S}_{\mathrm{NN}}}}=19.6 \mathrm{GeV}$ down to $3.0 \mathrm{GeV}$, corresponding to baryon chemical potential $\mu_{\mathrm{B}} \sim 200-720 \mathrm{MeV}$. The STAR BES II program is well defined. It aims for high precision studies of the type of phase diagram and location of critical point. Installed detector upgrades reduce systematic uncertainties and extend kinematic and PID range. RHIC upgrades increase luminosity.

The most interesting chapter in nuclear physics is around the corner!

\section{References}

[1] A. Bazavov, et al., Phys. Rev. D90 (2014) 094503

[2] F. Karsch et al., Nucl. Phys. Proc. Suppl. 129, (2014) 614

[3] M. Cheng et al., Phys. Rev. D79 (2009) 074505 and references therein 
[4] Y. Aoki et al., Nature 443 (2006) 675

[5] S. Ejiri, Phys. Rev. D78 (2008) 074507, E.S. Bowman and J.I. Kapusta, Phys. Rev. C79 (2009) 015202

[6] L. Adamczyk et al., STAR Collaboration, Phys. Rev. C96 (2017) 44904

[7] S. Wheaton et al., Comp. Phys. Comm. 180 (2009) 84

[8] J. Cleymans et al., Phys. Rev. C73 (2006) 034905

[9] A. Andronic et al., Nucl. Phys. A834, (20010) 237c

[10] J. Adams et al., STAR Collaboration, Nucl. Phys. A757 (2005) 102

[11] B. Abelev et al., STAR Collaboration, Phys. Rev. C79 (2009) 034909

[12] A. Andronic et al., Phys. Lett. B675 (2009) 312

[13] A.N. Tawfik et al., Int. J. Mod. Phys. E24 (2015) 1550067

[14] L. Adamczyk et al., Phys. Rev. Lett. 110 (2013) 142301, Phys. Rev. C88 (2013) 014902, Phys. Rev. C93 (2016) 014907

[15] S. Horvat et al., STAR Collaboration, Nucl. Phys. A956 (2016) 838, L. Adamczyk et al., STAR Collaboration, Phys. Rev. Lett. 121 (2018) 032301

[16] L. Adamczyk et al., STAR Collaboration, Phys. Rev. Lett. 116 (2016) 112302

[17] M.A. Stephanov, Phys. Rev. Lett. 107 (2011) 052301, B.J. Schaefer and M. Wagner, Phys. Rev. D85 (2012) 034027

[18] M. Asakawa et al., Phys. Rev. Lett. 85 (2000) 2072, V. Koch et al;., Phys. Rev. Lett. 95 (2005) 182301, M.A. Stephanov Phys. Rev. Lett. 102 (2009) 032301, C. Athanasiou et al., Phys. Rev. D 82 (2010) 074008

[19] L. Adamczyk at al., STAR Collaboration, Phys. Rev. Lett. 112 (2014) 032302

[20] X. Luo, POS CPOD2014 (2015) 019

[21] UrQMD - http://urqmd.org/

[22] M.Stephanov, J. Phys. G.: Nucl. Part. Phys. 38 (2011) 124147

[23] R. Holzmann, Nucl. Phys. A967 (2017)

[24] L. Adamczyk et al., STAR Collaboration, Phys. Rev. Lett. 113 (2014) 92301, Phys. Lett. B785 (2018) 551

[25] H. Stocker, Nucl. Phys. A750 (2005) 121; L.P. Csernai and D. Rohrich, Phys. Lett. B458 (1999) 454; J. Brachmann et al., Phys. Rev. C61 (2000) 24909; D.R. Rischke et al., Heavy Ion Phys. I, 309 (1995)

[26] J. Steinheimer at al., Phys. Rev. C89 (2014) 054913; V.P. Konchakovski et al., Phys. Rev. C90 (2014) 014903; W. Cassing et al., EPJ Web Conf. 95 (2015) 01004; Yu. B. Ivanov et al., Phys. Rev. C91 (2015) 024915; Y. Nara et al., Phys. Rev. C94 (2016) 034906

[27] L. Adamczyk et al., STAR Collaboration, Phys. Rev. Lett. 120 (2018) 062301

[28] J.Auvinen and H.Petersen, Phys. Rev. C88 (2013) 064908; D.Slanki et al., Phys. Lett. B720, (2013) 352 
[29] H.Liu et al., Phys. Rev. Lett. 84 (2000) 5488; P.Chung et al., Phys. Rev. Lett. 86 (2001) 2533

[30] C. Pinkenburg at al., Phys. Rev. Lett. 83 (1999) 1295

[31] M. A. Lisa et al., Phys. Rev. Lett. 84 (2000) 2798

[32] L. Ahle et al., Phys. Rev. C66 (2002) 054906

[33] E.Mount et al., Phys. Rev. C84 (2011) 014908; F. Retiere and M. A. Lisa Phys. Rev. 70 (2004) 044907; D. Rischke and M. Gyulassy, Nucl. Phys. A608 (1996) 479

[34] I. Pinayev et al., JACoW COOL2017 WEM22 (2018)

[35] STAR Collaboration Note 0644 (2015)

[36] STAR Collaboration Note 0666 (2016)

[37] STAR Collaboration, arXiv:1609.05102v1 (2016)

[38] X.Luo, Nucl. Phys. A956 (2016) 75

[39] J.Adam et al., STAR Collaboration, arXiv: 1903.0537 (2019)

[40] Y. Wu et al., STAR Collaboration, Nucl. Phys. A982 (2019) 899 